\title{
Hands-On Projects and Exercises to Strengthen Understanding of Basic Computer Engineering Concepts
}

\author{
Rohit Dua, John E. Seiffertt, Brian Blaha, Kapil Gupta, Venkat Satagopan, \\ Joe R. Stanley, Daryl Beetner, and Donald C. Wunsch \\ Department of Electrical and Computer Engineering \\ University of Missouri-Rolla \\ 1870 Miner Circle, Rolla, MO-65409, USA
}

\begin{abstract}
:
The Introduction to Computer Engineering course at the University of Missouri-Rolla provides a thorough understanding of basic digital logic analysis and design. The course covers: digital numbering systems, Boolean algebra, function minimization using Karnaugh maps (K-maps), memory elements, and sequential logic design. Students' grades are determined by their performance on homework assignments, quizzes, and in-class examinations. A laboratory course (optional for all but $\mathrm{EE}$ and $\mathrm{CpE}$ majors) supplements the lecture by providing experiments that include analysis and design using Mentor Graphics and FPGAs. While the laboratory is a very useful supplement to the lecture, almost half the students taking the lecture are not required to take the laboratory and there is not sufficient time in the laboratory schedule to introduce significant design elements. In Fall 2004, hands-on group projects, for all students, were introduced to the lecture course. The goal was for students to develop a more practical understanding and appreciation of hardware design and to improve motivation. Two projects were introduced that involve design of simple digital systems (based on practical applications), design optimization, and physical realization of the system using logic gates and/or memory elements. Two surveys, conducted during the semester, show the benefit of hands-on projects in gaining experience on basic digital hardware design.
\end{abstract}

\section{Introduction}

Introduction to Computer Engineering is a core course required to attain a Bachelor of Science (BS) degree in Computer or Electrical Engineering from University of Missouri-Rolla (UMR). The lecture course requires students to complete homework assignments, quizzes, two-to-three in-class or take home examinations, and a comprehensive final. The lecture course is supplemented by a laboratory where students can work with actual hardware. This laboratory has faced two problems. First, computer and electrical engineering majors are required to take the laboratory course along with the lecture course, but non-majors, such as Computer Science students, are only required to take the lecture. The computer science students constitute approximately one-third to one-half of the students in the lecture course. These students are missing out on many of the hands-on experiences available in the laboratory and their current curriculum does not have room to require the additional laboratory credit. Second, much of the time in the laboratory is spent learning new tools and reinforcing relatively simple concepts 
learned in the lecture. Students are required to perform experiments to verify theoretical concepts, but the choice of hardware to be used is rarely provided. Little time is available to institute a complete design project.

Instructors, in other universities, have improved introductory computer engineering course by adding components such as computer simulating programs and computer-aided engineering workstations to describe simple digital circuits and design philosophy [1,2], and FPGA based hardware prototyping system to build and simulate digital systems [3]. Modern design tools such as hardware descriptive languages (VHDL, ABEL) have made learning digital system design effective [4]. Such tools have been added to the laboratory component at UMR. To make the learning experience more effective and enjoyable for all students, a project component was added to the lecture course during the fall semester of 2004. Even though the laboratory provides a gateway into the world of hardware from the theoretical concepts learned in the lecture, a project component helps the student gain experience designing and building an application from the ground up. This exercise provides a means to verify design specifications and to experience the process of designing digital circuits. Obtaining experience using a project-based approach has been employed in an introductory programming course [5]. Projects offer an opportunity for students to explore hardware options and decide for themselves the optimal hardware needed to meet a design specification. A project also helps to meet ABET goals, as one of the ABET requirements is for students to demonstrate an ability to design a system, component or process to meet desired needs within realistic constraints [6].

The next section provides a description on the projects that were given to the Fall 2004 Introduction to Computer Engineering class at UMR. The effectiveness of materials was assessed through project reports, individual assessment feedback and student surveys. Results are summarized below. The description also includes a few observations and examples of designs. Conclusions are drawn and recommendations for future improvements are noted in the final section.

\section{Project Descriptions}

Two projects were assigned. The first was given after the instructor had covered minimization of functions, using Karnough-maps (K-maps) to obtain minimal Sum of Product (SOP), and Product of Sum (POS) expressions. Students were also able to convert these expressions to either the NAND or NOR based logic. Students were divided into groups of 4, such that at least one electrical or computer engineering student (thus enrolled in the lab) was in each group. Students enrolled for the laboratory course are more experienced with hardware, as well as the hardware simulation software available at our institution. The design problem used in the first project was as follows:

Problem description (Project I): Consider a missile defense facility. An EVAC alarm will go off, giving some time to evacuate, if the system has detected an incoming missile and the SAM (Surface to Air Missile) has failed to fire, or if the incoming missile has been detected and the SAM is fired but the SAM fails to intercept the incoming missile, or if the stored SAM becomes unstable under any condition. Design a logic circuit that will control the activation of the alarm. 
The first project was deliberately simple. The system has a maximum of four inputs and is based around a potentially real world application. The groups were required to accomplish the following goals:

1) Set up the problem. Determine the number of variables and their digital operation.

2) Determine the minimal SOP and POS equations using K-maps.

3) Compare different design options (minimal SOP, POS, NAND, NOR) based on available hardware, costs, etc. Give reasons for the choices used in your design.

4) Build the chosen circuit on a bread board using SSI components. Verify the circuit works by providing inputs using switches and observing outputs using LEDs.

Groups were required to purchase components from commercial vendors to build the project (however, for comparison purposes, in one section, the instructor provided the components). In order to assist students in purchasing components, the following information was provided:

- You will need to purchase the SSI chips to build your logic circuit. One possible source is http://www.fairchildsemi.com/ . The chips that you will be most interested in include:

○ Hex Inverter: 74AC04PC

- NAND2: 74AC00PC

- NOR2: 74AC02PC

- AND2: 74AC08PC

O OR2: 74AC32PC

O XOR2: 74AC86PC

All chips use DIP packages that are easy to mount on your breadboard. Other logic chips along with data sheets are available at http://www.fairchildsemi.com/products/logic/prod tree/logic function.html. These chips might be purchased from Digikey (www.digikey.com) or Radio Shack (http://www.radioshack.com/default.asp?site=top\%5Fhome).

- You will simulate built circuit using switches and LEDs. You can purchase them at very low cost. Both Digikey and Radio Shack have many LEDs available.

- You will need a DC battery to power your circuit. A 4.5 volt DC battery should be sufficient to power your circuit or you could use a 5 volt voltage regulator such as the LM7805.

Groups were required to submit a report that would include their design methodology, the derived logic expressions, and their final digital circuit. In some sections students were required to demonstrate the project in class, while in other sections students were instructed to submit their projects for assessment. For comparison purposes, in one section, the instructor had students submit their written final project reports. Then, students were requested to implement the final digital circuit using an instructor specified approach. In this case, students were required to implement the circuit using NAND-Based Logic. The instructor provided the students with the components for an in-class circuit implementation and demonstration. 
The second project was given out during the second half of the semester after the instructor had covered sequential network design. It was based on a modular design where students could build a digital system using higher order combinational components such as adders, comparators, latches, and counters. The project description was as follows:

Project Description (Project II): This project is an extension of project I. In project I you had four inputs: Incoming missile detected, SAM fired, missile intercepted, and SAM unstable. In your new project you will construct a logic circuit to generate the "missile intercepted" signal. Let's call it the MI (missile intercept) circuit. It will work as follows

- When an incoming missile is detected, the MI circuit will be toggled to an "enabled" state.

- When the MI circuit is enabled and a SAM is fired, a clock begins to count up.

- If the missile detected signal goes low while the clock count is less than 11 , then the MI circuit is disabled, the counter circuitry is reset, and the "missile intercepted" signal is set. If the missile detected signal is high when the count is greater than or equal to 11 , then the missile was not intercepted, the missile intercepted signal is cleared, and the EVAC alarm is sounded (as in project 1).

The student's goals were the same as for project 1 .

Since the project required a clock signal, the following information was also provided

- You can generate a clock signal in a couple of different ways. You can use a 555 timer (http://www.digikey.com/scripts/DkSearch/dksus.dll?Detail?Ref=77691\&Row=2 95800\&Site=US) that will "automatically" generate the clock signal or you may use a switch to manually generate the signal. If you use a switch, you will need to de-bounce the signal. To do so, you can purchase a debounced switch, or can build one using a capacitor and resistor or using an S-R latch.

- You will need to obtain or build a counter and a magnitude comparator to complete this project.

Figure 1 shows an example of a typical, completed, first and second project. Some of the designs had innovations such as a real time clocks and seven segment displays. 

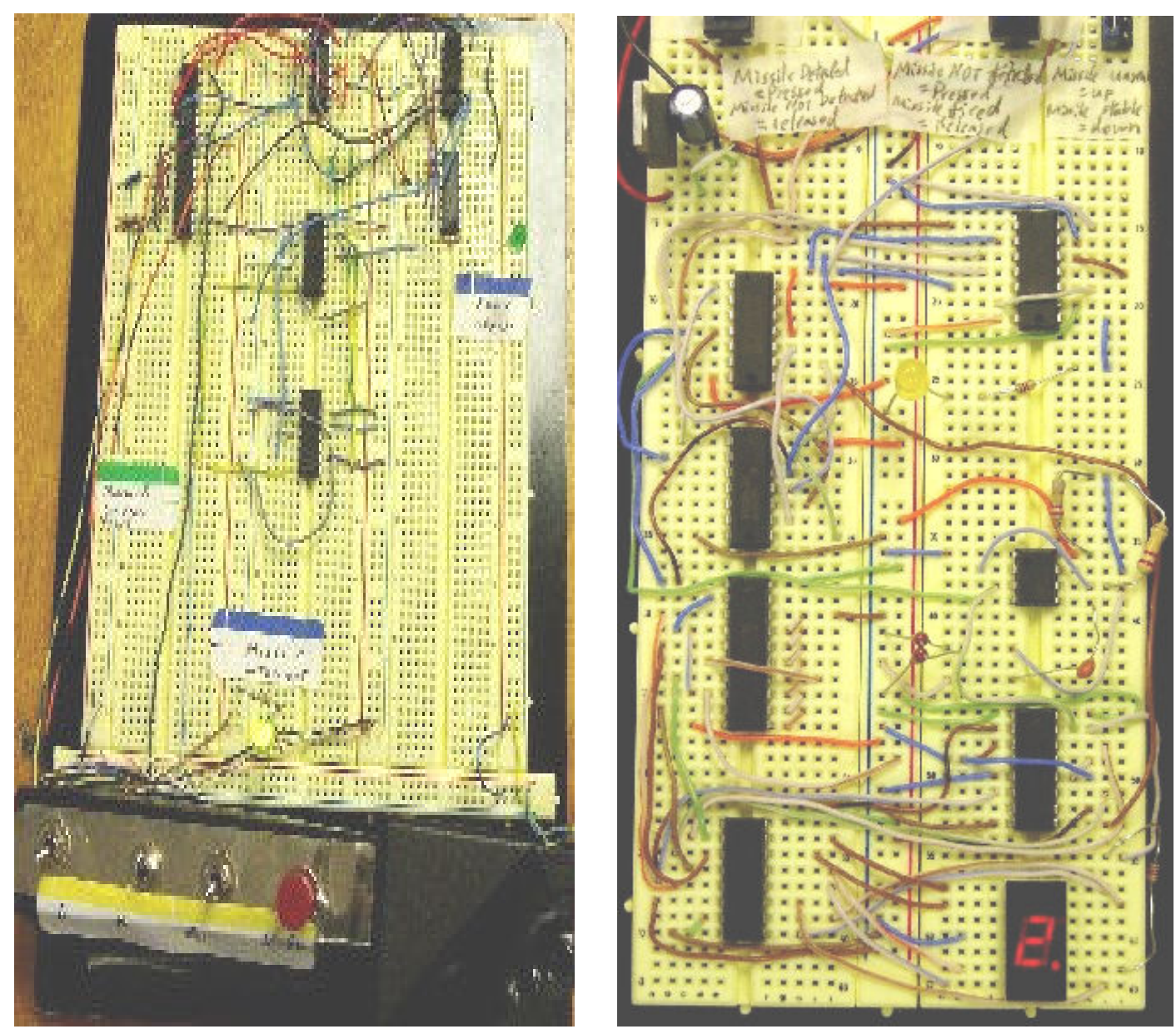

Figure 1. Typical projects 1 and 2. Students had the flexibility to include their own innovations, such as the seven segment display on the right.

\section{Surveys}

To assess the projects, surveys were conducted that included both rated and open ended questions. Table 1 shows the results and questions for project 1 . For rated questions, the scale ranged from " 1 " for strongly disagree to " 4 " for strongly agree. A high average shows a positive response. Ninety nine students responded to the first survey. 
Table1: Survey results for Project 1

\begin{tabular}{|c|c|c|c|c|c|}
\hline \multirow{3}{*}{ Question No. } & \multicolumn{4}{|c|}{ Survey 1} & \multirow[b]{3}{*}{4} \\
\hline & \multirow[t]{2}{*}{ Average } & \multicolumn{3}{|c|}{ Scale } & \\
\hline & & 1 & 2 & 3 & \\
\hline 1 & 3.061 & 4 & 15 & 51 & 29 \\
\hline 2 & 3.242 & 1 & 13 & 46 & 39 \\
\hline 3 & 3.040 & 5 & 23 & 34 & 37 \\
\hline 4 & 3.010 & 6 & 24 & 32 & 37 \\
\hline 5 & 2.717 & 8 & 32 & 39 & 20 \\
\hline 6 & 2.778 & 6 & 34 & 35 & 24 \\
\hline 7 & 2.657 & 13 & 31 & 32 & 23 \\
\hline 8 & 2.949 & 4 & 30 & 32 & 33 \\
\hline
\end{tabular}

Questions

1. I found the project to be an enjoyable experience

2. The problem specification was helpful for this kind of project

3. The project gave me a better appreciation of the course material

4. The project gave me a better understanding of hardware design procedures

5. My confidence level has improved after doing this project

6. I am motivated now to learn more about digital circuit design

7. I learned a lot about collaboration (teamwork)

8. I now have a better understanding of selecting the right components for a digital design

Several observations can be made as a result of this survey. Most of the students found the project to be an enjoyable experience. Also, most students found the problem specification adequate for this kind of project. More than half the students found the project gave them a better appreciation of course material and a better understanding of hardware design procedures resulting in motivation to learn more about digital circuit design. Some of the groups had problems with collaborations. The rating on question number 7 reflects this. But, solutions to problems in teamwork were solved with meetings with all the group members. Of the four sections, groups from three sections were required to purchase components from vendors. This exercise proved to be an expensive affair. Even though the chips themselves cost a few dollars, shipping is expensive resulting in increased budgetary requirements. Nevertheless, students attained the experience to select the right components including the type of chips needed to build the project. Comments made by students were used to help improve the second project.

A similar survey was conducted at the end of the project 2. Table 2 depicts the results from this survey. The goal of the survey was to find out whether there was any improvement in ratings from project 1 . Eighty three students responded to the second survey. 
Table 2: Survey results for project 2

\begin{tabular}{cccccc}
\hline \multirow{2}{*}{ Question No. } & Average & \multicolumn{2}{c}{ Survey 2 } & \multicolumn{2}{c}{ Scale } \\
& & 1 & 2 & 3 & 4 \\
\hline 1 & 3.024 & 4 & 17 & 38 & 24 \\
2 & 2.963 & 3 & 18 & 44 & 18 \\
3 & 3.110 & 8 & 11 & 31 & 33 \\
4 & 3.012 & 4 & 17 & 39 & 23 \\
5 & 2.780 & 8 & 24 & 32 & 19 \\
6 & 2.659 & 10 & 24 & 36 & 13 \\
7 & 3.000 & 11 & 14 & 25 & 33 \\
8 & 2.878 & 6 & 20 & 38 & 19 \\
\hline
\end{tabular}

Question

1. I found the project to be an enjoyable experience

2. The problem specification was helpful for this kind of project

3 . The project gave me a better appreciation of the corresponding course material

4. The project gave me a better understanding of modular and sequential hardware design procedures

5. My confidence level improved after doing this project

6. I am motivated now to learn more about advanced topics and take higher level courses in Computer Engineering

7. Collaboration (teamwork) with my team members has improved since the first project

8. I now have a better understanding of selecting the right components for a digital design

Comparing the ratings of the two surveys, following conclusions can be drawn from the second survey.

- Most students found the project to be an enjoyable experience.

- Some students found the problem specification a bit confusing, which led to a dip in the rating for the second question. Appropriate changes will be made to next semester's project to help clear up this confusion.

- The second project gave a better appreciation for the relevant course material and modular design procedures for most of the students, thereby, increasing their confidence level.

- Collaboration between students in groups improved during the execution of the second project. This was brought about by sorting out issues some of groups had during the first project.

- Most students now have a better understanding of selecting the right components for projects and are motivated to take advanced courses in Computer Engineering. 


\section{Conclusions and Future Improvements}

A project provides an opportunity, for students taking the Introduction to Computer Engineering course to design and build simple digital circuits from the ground up. Two projects were executed, the second project being the extension of the first. The overall student response was positive regarding project specifications, and relevance to course topics. Students gained experience interacting within groups to complete a technical project. The surveys conducted reflect this to some extent. A question that arises is did students develop a more practical understanding and appreciation of hardware design. We need a better measure of their understanding. This can be achieved by measuring student performance on a test or future projects against some control group. For now, we, as instructors, can comment on observations made during the semester. Some groups were eager to build a complex second project from a deliberately simple first project. Student interaction, in groups, improved over the semester; this will help them perform better in future project assignments. We aim at obtaining a better assessment, by conducting a survey during the next level course in computer engineering, Digital Systems Design. We could compare student performance between those who did and did not do our projects.

Several improvements to the projects are possible. Depending on budget constraints, an attempt will be made in future semesters to provide a cache of SSI chips and other relevant components for loan to students to help make projects less expensive. If more time is available, a variety of similar projects can be developed, allowing different groups to implement different projects, possibly projects that will all be used together to make a greater whole.

\section{References}

[1] Y.-Y.J. Leung, "The use of computer-aided engineering workstation in digital-circuit design laboratory," IEEE Transactions on Education, 32 (3), pp. 375-377, August 1989.

[2] P. Corsini, and L. Rizzo, "SSCSSC: a tool for the teaching of digital circuits," IEEE Transactions on Education, 34 (1), pp. 70-75, February 1991.

[3] A. Zemya, A. Trost, and B. Zajc, “A rapid prototyping environment for teaching digital logic design,” IEEE Transactions on Education, 41 (4), pp. 8, November 1998.

[4] Adnan Shaout, N. Narasimhamurthi and P. Watta, "Enhancing the digital systems courses with modern design tools and practices," Proceedings of the ASEE conference, Albuquerque-new Mexico, paper no. 348, June 24-27, 2001

[5] D. Davenport, "Experience using a project-based approach in an introductory programming cours.e," IEEE Transactions on Education, 43 (4), pp. 443-448, November 2000.

[6] http://www.abet.org/images/Criteria/E001\%2005-06\%20EAC\%20Criteria\%2011-17-04.pdf, pp. 9-10.

\section{Biographies}

Rohit Dua is a PhD candidate in the Department of Electrical and Computer Engineering. His research interests include neural networks applied to smart structures and pattern recognition of skin cancer profiles. He is a member of IEEE, and Eta Kappa Nu. 
John E. Seiffertt is currently a PhD student in Department of Electrical and Computer Engineering.

Brian Blaha is a PhD Candidate at UMR. He teaches an introductory hardware and software lab to sophomores and juniors. His research focuses on evolutionary computation as applied to games and NP-complete problems.

Kapil Gupta received B. E. in Electronics and Telecommunication Engineering from Baba Saheb Naik College of Engineering, Pusad affiliated to Amravati University, India in May 2001. He received his M.S. in Electrical Engineering from the University of Missouri, Rolla in May 2004, with an emphasis in signal processing and communications.

Venkat Satagopan is currently a PhD Candidate in the Department of Computer Engineering.

R. Joe Stanley is an assistant professor of ECE at UMR. Prior to UMR, he served as Principal Investigator for the Image Recognition program at Systems \& Electronics, Inc. in St. Louis, MO. He received his Ph.D. degree in Computer Engineering and Computer Science from the University of Missouri-Columbia in 1998. His research interests include signal and image processing, pattern recognition, and automation.

Daryl Beetner is an associate professor of ECE at UMR. He received his B.S. degree in Electrical Engineering from Southern Illinois University at Edwardsville in 1990. He received an M.S. and D.Sc. degree in Electrical Engineering from Washington University in St Louis in 1994 and 1997, respectively. He conducts research on a wide range of topics including electrocardiology, skin cancer detection, humanitarian demining, and electromagnetic compatibility.

Donald C. Wunsch, M.K. Finley Missouri Distinguished Prof. of ECE at UMR, was previously with Texas Tech, Boeing, Rockwell Intl., and Intl. Laser Systems. He is INNS President, an IEEE Fellow, NSF CAREER Awardee, has over 200 publications, attracted over $\$ 5$ million funding, and produced $8 \mathrm{Ph} . \mathrm{D}$.'s. 